\title{
Long-term X-ray Variability of NGC 4945
}

\author{
Amara Miller \\ Science Undergraduate Laboratory Internship Program \\ University of California, Davis \\ Stanford Linear Accelorator Center (SLAC) \\ Stanford, CA
}

August, 2007

Prepared in partial fulfillment of the requirement of the Office of Science, Department of Energy's Science Undergraduate Laboratory Internship under the direction of Grzegorz Madejski, a member of the Kavli Institute at the Stanford Linear Accelerator Center.

Participant:

Signature

Research Advisor:

Signature 


\section{TABle of Contents}

Abstract ............................... i

1. Introduction . . . . . . . . . . . . . . . . . . . . 1

2. Materials and Methods . . . . . . . . . . . . . . 3

3. Results . . . . . . . . . . . . . . . . . . 8

4. Discussion and Conclusion . . . . . . . . . . . . 10

Acknowledgments ..................... . . . 11

References . . . . . . . . . . . . . . . . . 12

Figures ........................... . . . . 14 


\begin{abstract}
Long-term X-ray Variability of NGC 4945. AMARA MILLER (University of California Davis, Davis, CA 95616) GRZEGORZ MADEJSKI (Stanford Linear Accelerator Center, Stanford, CA, 94305).
\end{abstract}

Though short-term X-ray variability has been studied for the active galaxy NGC 4945, long-term studies promise to contribute to our understanding of the processes involved in accretion onto supermassive black holes. In order to understand the relationship between black hole mass and breaks in the power spectral density (PSD), the long-term X-ray variability of NGC 4945 was studied over the energy range 8-30 keV. Observations occurred over the year 2006 using the Rossi X-ray Timing Explorer. The data was reduced using the package FTOOLS, most notably the scripts Rex and faxbary. Light curves were produced and a PSD was obtained using a Fast Fourier Transform algorithm. Preliminary studies of the light curve show greater X-ray variability at higher frequencies. This result complements previous studies of NGC 4945 by Martin Mueller. However, the PSD produced must go through further study before accurate results can be obtained. A way to account for the window function of the PSD must be found before the behavior at lower frequencies can be studied with accuracy and the relationship between black hole mass and the break in NGC 4945's PSD can be better understood. Further work includes exploration into ways to subtract the window function from the PSD, as well as a closer analysis of the PSD produced by averaging the data into logarithmic bins. The possibility of a better way to bin the data should be considered so that the window function would be minimized. 


\section{INTRODUCTION}

Most galaxies in the universe show little activity beyond what would be expected for a collection of stars and gas. However, a small percentage of observed galaxies (called active galaxies) show extreme activity well above what is considered normal. The accepted theory today is that an active galaxy is powered by accretion of galactic gases onto a

supermassive black hole [1], giving it the name of an active galactic nucleus (AGN) [2]. By using Hubble's law in conjunction with the measured redshift, the distance can be obtained and it is clear the luminosity of these objects is unusually high. The mass of the black hole can be constrained from the luminosity and thus the Eddington limit. The rapid variability of X-ray radiation implies that the size of the nucleus is extremely small for the amount of mass present, supporting the theory of black hole accretion. The mass of a black hole is expressed in units of solar mass. One solar mass is equivalent to the mass of the sun, $\sim 2 \times 10^{30} \mathrm{~kg}$, and is expressed by the symbol $M_{\odot}$. Compared to a smaller black hole of mass $1.0-3.0 M_{\odot}$ (such as those inferred to exist in the binary systems of the galaxy), a supermassive black hole can be as large as $10^{9} M_{\odot}$.

According to the model of an accreting supermassive black hole, instabilities within the material orbiting the galaxy begin to redistribute angular momentum and cause matter to spiral inward, forming an accretion disk about the nucleus. The energy released in this process can be emitted as electromagnetic radiation within a wide band range, including X-ray wavelengths. The current model of this process explains the presence of X-ray emission through inverse Compton scattering. This occurs when lower energy photons are scattered to higher energies when they interact with nearly-relativistic electrons in the hot gaseous material of the accretion disk. The cold material farther from the nucleus radiates as emission lines. Taking into account these effects, the observed broad- 
band spectrum from AGNs can be explained. However, the exact processes involved are not fully understood, and the black hole model is not yet completely confirmed. Despite difficulty in understanding AGN, they display strong cosmological evolution which furthers the study of the early universe. Most of the knowledge we have about the intergalactic medium comes from the study of absorption lines in the spectra of distant quasars [2], one class of AGNs.

Both light curves and spectra are used to study AGNs. A light curve is a graph of the light intensity of an observed object as a function of time. In this project the term spectrum refers to a power spectral density (PSD), a graph of the variability amplitude of the light curve plotted against the frequency of variability. This amplitude often takes the shape of a powerlaw. However, at a certain point this powerlaw must break into a different structure since a single power law is divergent mathematically. According to [3] and [4], there is a strong correlation between the black hole mass of an AGN and its associated PSD break amplitude. The break amplitute is where the single power law description is no longer applicable to the data. This relationship is not fully understood, and it is the goal of this paper to explore this correlation using data obtained for NGC 4945.

NGC 4945 is a nearby, edge-on starburst galaxy with an active nucleus of the Seyfert type 2 classification. In the first few X-ray studies, the data revealed a heavily obscured, strong, hard X-ray source above $10 \mathrm{keV}$. Because the circum-nuclear material is partially optically thick to electron scattering, the absorbing column is one of the largest ( $10^{24} \mathrm{~cm}^{-2}$ ) that still allows a direct view of the nucleus at hard x-ray energies (see Figures $4 \& 5$ ). NGC 4945 is a megamaser source, implying the edge-on geometry mentioned previously. It is one of only a few AGNs for which the black hole mass can 
be constrained (at $\sim 1.4 \times 10^{6} M_{\odot}$ ) from detailed mapping of the megamaser spots [5]. It has been determined that this object has a high variability on short time scales, but in order to determine the properties of this active galaxy more accurately, the variability on longer time scales is studied below using data collected by the Rossi X-ray Timing Explorer (RXTE). This project will supplement data that has been obtained and studied in previous experiments. Because of the unique position of knowing the black hole's mass reliably, the data collected will further our understanding of the relationship between the break in the PSD of an AGN and it's black hole mass [6]. It will also contribute to the study of the structure of AGNs, as well as our understanding of how and why X-ray radiation is produced.

The low-earth orbit satellite $R X T E$ features unprecedented time resolution combined with a moderate spectral resolution and broad-band X-ray sensitivity, making it ideal for studying the variability of cosmic X-ray sources. It carries two instruments, both of which will be used in this study: the Proportional Counter Array (PCA) and the High Energy X-ray Timing Experiment (HEXTE) [7]. More about the data collected from the Rossi XTE is discussed at greater length below.

\section{Materials AND Methods}

In order to fully analyze the data from $R X T E$, light curves and spectra had to be produced. However, a PSD of all the observations of NGC 4945 was not immediately available from the data collected by $R X T E$, so various reduction processes took place prior to the final analysis. In addition, there are inherent limitations associated with the duration and sampling rate of the observation that restrict the range of frequencies that can be analyzed using a Fast Fourier Transform (FFT) algorithm on the spectrum. Data 
reduction scripts used were obtained from the HEASARC website, including the packages FTOOLS (a data analysis package for astronomical applications) and XANADU (a suite of spectral, timing, and image analysis programs). The file format used was the HEASARC standard Flexible Image Transport System (FITS).

Every four days over the course of the year 2006 NGC 4945 was observed for $\sim 40$ minutes. In this project both PCA and HEXTE data were used to study the energy range from 8-30 keV. The PCA on RXTE contains five proportional counters referred to as PCUs 0-4, each with one propane layer and 4 Xenon layers operating within an energy range of 2-60 keV [7]. PCUs 0,3 , and 4 were not on during the entire observation, and as of December 25, 2006, PCU 1 developed a leak in it's propane layer. Because the background data for PCU 1 would not be accurate for all the data collected, as well as the fact that it was on for only a minimal amount of time during data collection, PCU 1 data was not used in the analysis. For these reasons it was necessary to ignore data from PCUs $0-1$ and 3-4 and only use PCU 2 to extract light curves and spectra from NGC 4945.

HEXTE consists of two clusters of 4NaI/CsI phoswich scintillation detectors that are sensitive to X-rays from $15-250 \mathrm{keV}[8]$. Because this energy range does not cover the lower energy X-rays within our desired data set, HEXTE was mainly used to check the data and the reduction process. Data from cluster 1 of the HEXTE system was used when producing light curves and spectra.

PCA data were reduced according to the restrictions discussed above using standard RXTE analysis tools, most notably Rex, a component of FTOOLS. Rex is script designed to run through the basic data reduction of PCA and HEXTE for multiple observations. Rex initially read the index files in the database structure and proceeded to filter the 
data. After the filtering process was complete, the program then produced a model background file which was subtracted from the results [9]. The background file used in analysis of PCA data was the most recent available from the HEASARC website for faint sources, pca_bkg_cmfaint17_eMv20051128.mdl. The energy channels analyzed were 18-73, the equivalent of 8-30 keV (the energy range desired in this study). Rex was then run on the Cluster 1 HEXTE data for the 7-41 channel range, corresponding to $~ 12-100$ $\mathrm{keV}$. There were $\sim 150$ different observations of NGC 4945 over the course of 2006, and Rex produced a combined light curve and raw spectrum for all observations. These light curves and spectra were then analyzed with the tools lcurve and xspec to check the data for accuracy (i.e., no obvious spikes or instabilities).

Because the observations of NGC 4945 occurred over such a long time frame, it was necessary to consider the revolution of the earth about the sun. This revolution places the satellite at a greater distance from the source half the year, and the additional light travel time must be accounted for in the results. The correction is often called the Barycenter Correction, a procedure that has been built into a tool called faxbary, part of the HEARSARC software FTOOLS. Before running faxbary, a list file was created containing all orbit files that covered the entire length of the observation (Days 4450 to 4875). Faxbary was then applied to both HEXTE and PCA data using the most accurate clock corrections in the file tcd.dat. After running the script, a copy of the original light curve file with an additional column, BARYTIME, was created. This additional column contained the corrected timing data for the observation.

In order to produce a PSD using a Fast Fourier Transform algorithm, it was necessary to place the timing data into consecutive time bins. The desired result would bin the data collected from each day into an individual "newbin." Because the satellite only 
collected data on NGC 4945 approximately every 4 days, and on each of those days had multiple $16 \mathrm{~s}$ observations, this binning took three steps. Initially, a basic $\mathrm{C}$ program was written using the FITSIO library of $\mathrm{C}$ subroutines for reading and writing data files in FITS data format. This program took the barycentric corrected times and subtracted the initial time of the observations from each value. It then divided the resulting value by $16 \mathrm{~s}$ and rounded the remainder to the nearest integer, creating a list of the equivalent $16 \mathrm{~s}$ binned times.

Another C program was written to output the start and end row/bin numbers, as well as the number of $16 \mathrm{~s}$ observations and the gap size between observations that occurred every 4 days. This table was analyzed and a new rebinning time of $16200 \mathrm{~s}$ (and offset of 137) was determined. This new rebinning time was able to place observations that were obtained on the same day into the same interval. A program was written to place the $16 \mathrm{~s}$ bins calculated in the previous step into these larger bins corresponding to the sampling which took place $\sim 3-4$ days. The larger bins were intervals within which the count rates were averaged. The error for the count rate was also calculated, using the formula

$$
c=\frac{1}{N} \sqrt{\sum_{i}^{N}\left(a_{i}\right)^{2}}
$$

where $c$ is the averaged error for a newbin, $N$ is the number of data entries averaged, and $a_{i}$ is the $i^{\text {th }}$ data entry for the error. Thus the output of the final rebinning was a fits file containing three rows: the averaged count rate per newbin, the error per newbin, and the time corresponding to the newbin. The time was calculated using $t_{n}=n \Delta$, where $\Delta$ is the time step (the rebinning time $16200 \mathrm{~s}$ multiplied by $16 \mathrm{~s}$ ) between each $n^{\text {th }}$ entry.

A Fast Fourier Transform algorithm was run on the final rebinned data and used to create a PSD for NGC 4945. A physical process can be described as some quantity 
as a function of time, $h(t)$, or as the process's amplitude as a function of frequency, $H(f)$. A Fourier Transform is a mathematical expression relating these two different representations of the same process. The equations for a Fourier Transform are

$$
\begin{gathered}
H(f)=\int_{-\infty}^{\infty} h(t) e^{2 \pi i f t} \mathrm{~d} t \\
h(t)=\int_{-\infty}^{\infty} H(f) e^{-2 \pi i f t} \mathrm{~d} f
\end{gathered}
$$

In a process involving complex values (such as a wave function) $H(f)$ would differ throughout the frequency range of $-\infty<f<\infty$. Because the discrete values dealt with in this project, $H(f)<0$ is a reflection about the y axis of $H(f)>0$. The algorithm called realft found in Numerical Recipes [10] was used, since the data analyzed was both discrete and real. This algorithm took the averaged count rate for the rebinned times and produced as output the transformed power values. These values corresponded to both the real and imaginary components for a corresponding frequency. To obtain the amplitude, which was the desired result, the program then squared both the real and complex components and added them together.

As stated previously, there are inherent limitations associated with the sampling rate of the observation that restrict the range of frequencies that can be analyzed using a Fast Fourier Transform (FFT) method on the spectrum. A window function is a set of data which has the same sampling as the data used to obtain the desired PSD, and so is able to show the inherent limitations associated with the specific sampling process. To check the results of the PSD, a window function of the rebinned data was produced by creating a parallel set of data that had gaps in the same locations as the PCA count rates and the value one in every location that a value existed in the actual data set. This correlated set 
of values was then run through an FFT to produce a new fits file that was used to graph a "blank" PSD showing the inherent errors in a PSD with the same data sequence.

\section{Results}

As described above, after running Rex the light curves and spectra were then analyzed with the tools lcurve and xspec to check if the data appeared accurate. Figure 1 and Figure 2 show the two light curves produced for PCA and HEXTE data using lcurve. Both have a newbin time of 86400 seconds. The average count rate for data collected by the PCA is 1.19 counts/s. The average count rate for data collected by HEXTE is slightly smaller at 1.02 counts/s. The count rates of both the PCA and HEXTE data do not vary significantly from the average count rate over the course of the year. There is a data gap after the first three data points up to $\sim 6 \times 10^{6} \mathrm{~s}$. The data are then continuous, though there are regular, periodic, linear increases in the count rate in Figure 1. These periodic increases are harder to see in Figure 2. The error for the HEXTE data (Figure 2) is greater than that seen on the PCA data (Figure 1).

Figure 3 compares the two light curves for PCA and HEXTE data. Plotting one against the other, the mean line nearly has a slope of one, indicating that the two sets of data correlate, paralleling each other in shape. Errors on this graph are not included, but are $\sim 0.1-0.2$, or $10 \%$. With errors included, it is possible the two sets of data correlate better than shown. The fact that they correlate in Figure 3 implies that the data reduction process was sucessful and that there were no unexpected jumps or discontinuities.

The raw data obtained from Rex was also analyzed with xspec to see if the energy spectrum was consistent with previous observations of NGC 4945. The model used was 
a powerlaw, plus a Gaussian emission line (due to the presence of iron, commonly seen in AGN), plus a photoelectric absorption to which there was added another powerlaw (to account for nearby weak x-ray sources that were also included in the field of view). This was run on both the PCA and HEXTE data, as shown in Figures 4 and 5. This model was very rough with a chi-squared was 367.83 for 82 bins, or the reduced chi-squared was 4.9706 for 74 degrees of freedom. The large chi-squared is most likely due to the poorly modeled low energy region, in which there are fluctuations outside of the predicted model.

Figure 4 shows the modeled data along with the residuals of the model. The PCA data is the first unbroken line of data covering the range $3-30 \mathrm{keV}$. The second line in the graph from 30-80 keV is of the HEXTE data, which observes at higher energies. Most of the residuals are within the 3 to $7 \mathrm{keV}$ range where there are fluctuations in the data. These low energy residuals are probably due to inadequately modeling of the host galaxy, but are not relevant to the study of NGC 4945 since we are interested in higher energies. Figure 5 is graphed in a logarithmic space. It is clear from this graph that the HEXTE and PCA data closely follows the model, with no obvious breaks at energies higher than $7 \mathrm{keV}$. As touched on earlier, there are minor fluctuations in the low energy range of 3 to $7 \mathrm{keV}$.

Figure 6 shows the PSD obtained from the FFT transform. It is graphed on a logarithmic scale with power and as a function of frequency. From the beginning of the graph to $5 \times 10^{-7} \mathrm{~Hz}$ the power jumps up and down, starting very large and oscillating in sharp peaks. From $5 \times 10^{-7} \mathrm{~Hz}$ to the end of the graph the data also fluctuates up and down in spikes, but it is less sporadic and there is a clear linear increase with a slope of $\sim 1$. Figure 7 shows the Window Function for the PSD in Figure 6. The window function 
shows similar jumps in the data from the beginning of the graph to $\sim 5 \times 10^{-7} \mathrm{~Hz}$, after which the oscillations begin to level off and show a constant average for the remainder of the graph. This behavior is unusually sporadic. It was expected that the data set would begin large, drop suddenly after the initial data points, and then oscillate in a series of jumps until it reached zero. Such a graph would accurately represent what would appear should the sampling be a gap followed by a large block of data. This window function is unexpected, with uneven jumps and no sudden drop.

\section{Discussion and Conclusion}

According to Figure 1, the average count rate does not change significantly over the course of the observation, implying that there is little variability on long time scales. There does appear to be variability on smaller time scales of approximately several weeks to a month as indicated by the linear increases in count rate at periodic intervals. This is promising, for it would imply the data collected correlates with previous studies. However, looking at Figure 6 there is little indication of variability at shorter time scales. Though there is a linear increase at higher frequencies, it is on the order of $f^{1}$, which is an unexpected

result. It is expected that the PSD should portray a $f^{-1}$ decrease, or no rise at all. When comparing Figure 6 to its window function in Figure 7, it is possible that the uneven power at lower frequencies is being reflected onto higher frequencies, and thus causing a linear increase to appear. The window function also shows similar jumps in power at lower frequencies, implying that these unusual attributes of the PSD are also a product of the way the data set is sampled. This makes it impossible to analyze the PSD until a way can be developed to account for the window function.

Because the PCA and HEXTE data correlate (shown in Figure 3), it is inferred that 
the variability of NGC 4945 is intrinsic rather than extrinsic. PCA and HEXTE detectors work within different energy ranges, and if the light curves correspond to each other it implies that the energy spectrum does not change appreciably, even over large changes in the intensity of the source. Unlike intrinsic variability, extrinsic variability would be caused by some object, such as a cloud of gas, interfering with the light coming from the active galaxy NGC 4945 and mimicking a variability intrinsic to the source. Were this the case, if there was an energy change the intensity of HEXTE and PCA would not necessarily correlate. Because it is shown that they do, it can be inferred that it is much more likely for the variability to be intrinsic to the source.

Studying time scales up to the full year of the observation proved to be more difficult than expected since the window function for the data was too rough to enable an accurate study of variability at lower frequencies. Further study will not be possible until a way is discovered to account for the window function and subtract it from the data. At present, work is being done to look into this possibility and analyze the window function more thoroughly. Further work should include a closer analysis of the PSD produced by averaging the data into logarithmic bins. The possibility of a better way to bin the data should be considered so that the window function would be minimized. This could include multiplying the light curve by a window function before implementing a FFT, as was done in [11]. Finally, if it is possible to obtain clearer results, a comparison between the long-term and short-term variability of NGC 4945 should take place so that the PSD break can be determined more accurately. With this knowledge, it will be possible to investigate the relationship between the PSD break of NGC 4945 and the black hole mass. 


\section{ACKNOWLEDGMENTS}

This research was conducted at the Stanford Linear Accelerator Center (SLAC), in Menlo Park, CA. I would like to thank my mentor, Greg Madejski, for his patience at my constant questions as well as for his wonderful advice and support. Special thanks go to Martin Mueller, who helped me understand the sequence of steps needed as well as directed me to various programming resources and excellent advice. Special thanks goes to all those involved at the SULI program at SLAC, especially Program Administrator Farah Rahbar and Program Director Steve Rock. Lastly, I would like to thank the Department of Energy for the opportunity to participate in this research program and the incredible learning experience I have had.

\section{REFERENCES}

[1] Frank H. Shu,The Physical Universe: An Introduction to Astronomy, Mill Valley: University Science Books, 1982.

[2] Julian H. Krolik, Active Galactic Nuclei: From the Central Black Hole to the Galactic Environment, Princeton University Press, 1999.

[3] Philip Uttley and Ian McHardy, "A Brief Review of Long-term X-ray and Optical Variability in Radio-quiet AGN." Feb. 2004.

[4] A. Markowitz et. all, "X-ray Fluctuation Power Spectral Densities of Seyfert 1 Galaxies." The Astrophysical Journal, March 2003.

[5] Greg Madejski et. all, "Structure of the Circumnuclear Region of Seyfert 2 Galaxies 
Revealed by Rossi X-ray Timing Explorer Hard X-ray Observations of NGC 4945." The Astrophysical Journal, May 2000.

[6] Martin Mueller et. all, "The X-ray Variability of NGC 4945." Dec. 2003.

[7] RXTE Guest Observer Facility, "The Rossi X-ray Timing Explorer Mission." 2007, Available at HTTP: http://rxte.gsfc.nasa.gov/docs/xte/xtegof.html.

[8] Philip Blanco, "The High Energy X-ray Timing Experiment." 1996, Available at HTTP: http://rxte.gsfc.nasa.gov/docs/xte/HEXTE.html.

[9] RXTE Guest Observer Facility, The RXTE Cook Book. 2007, Available at HTTP: http://heasarc.gsfc.nasa.gov/docs/xte/recipes/cook_book.html.

[10] William H. Press et. all, Numerical Recipes: The Art of Scientific Computing, New York: Cambridge University Press, 2007.

[11] Rick Edelson and Kirpal Nandra, "A Cutoff in the X-ray Fluctuation Power Density Spectrum of the Seyfert 1 Galaxy NGC 3516." The Astrophysical Journal, April 1999. 


\section{FiguRES}

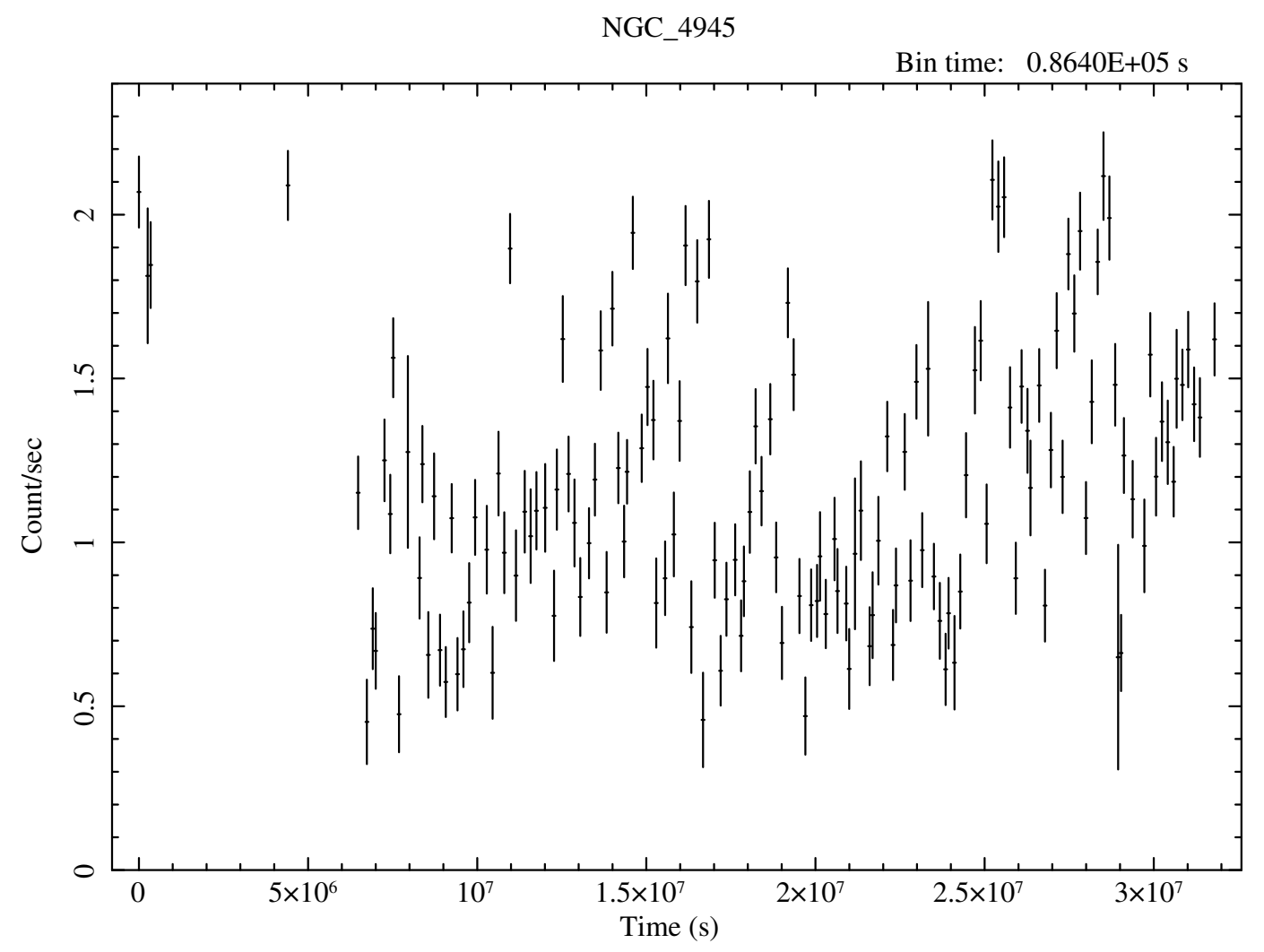

Start Time 13825 23:15:59:562 Stop Time 14193 23:15:59:562

Figure 1: RXTE light curve for NGC 4945 in the $8-30 \mathrm{keV}$ band produced using PCA data. This graph was created using lcurve with a newbin time was $86400 \mathrm{~s}$. Error bars are shown. 


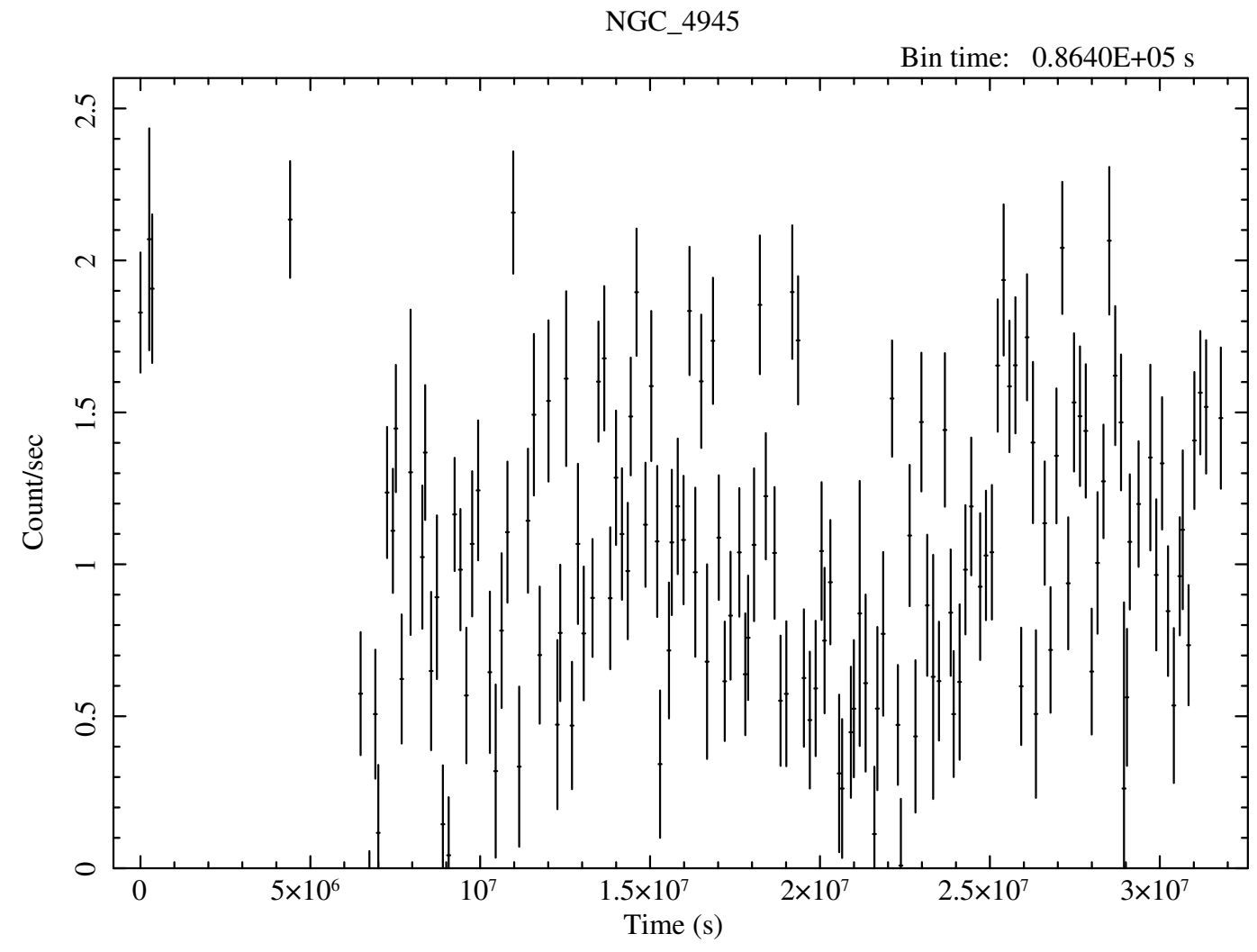

Start Time 13825 23:16:31:562 Stop Time 14193 23:16:31:562

Figure 2: RXTE light curve for NGC 4945 in the $12-100 \mathrm{keV}$ range produced from HEXTE data. This graph was also created using lcurve with a newbin time of $86400 \mathrm{~s}$. Note that the shape of the HEXTE light curve reflects that of the PCA light curve. 


\section{Light Curve Comparison}

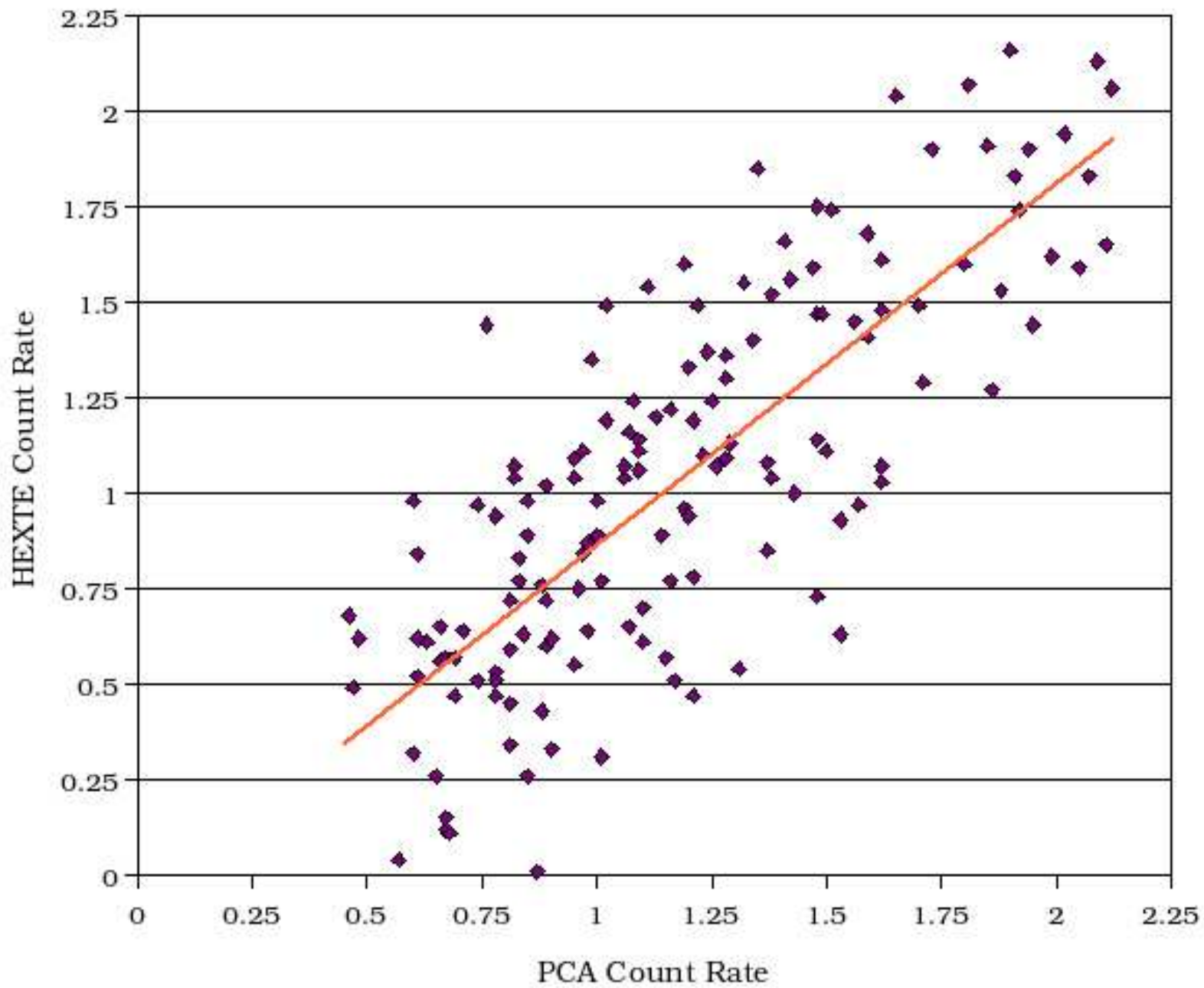

Figure 3: This graph plots the PCA count rate against the HEXTE count rate for the observed object, NGC 4945. Both count rates were obtained from light curves produced by the script Rex, part of FTOOLS. The mean line has a linear slope, indicating that the PCA and HEXTE data closely parallel each other in shape. 


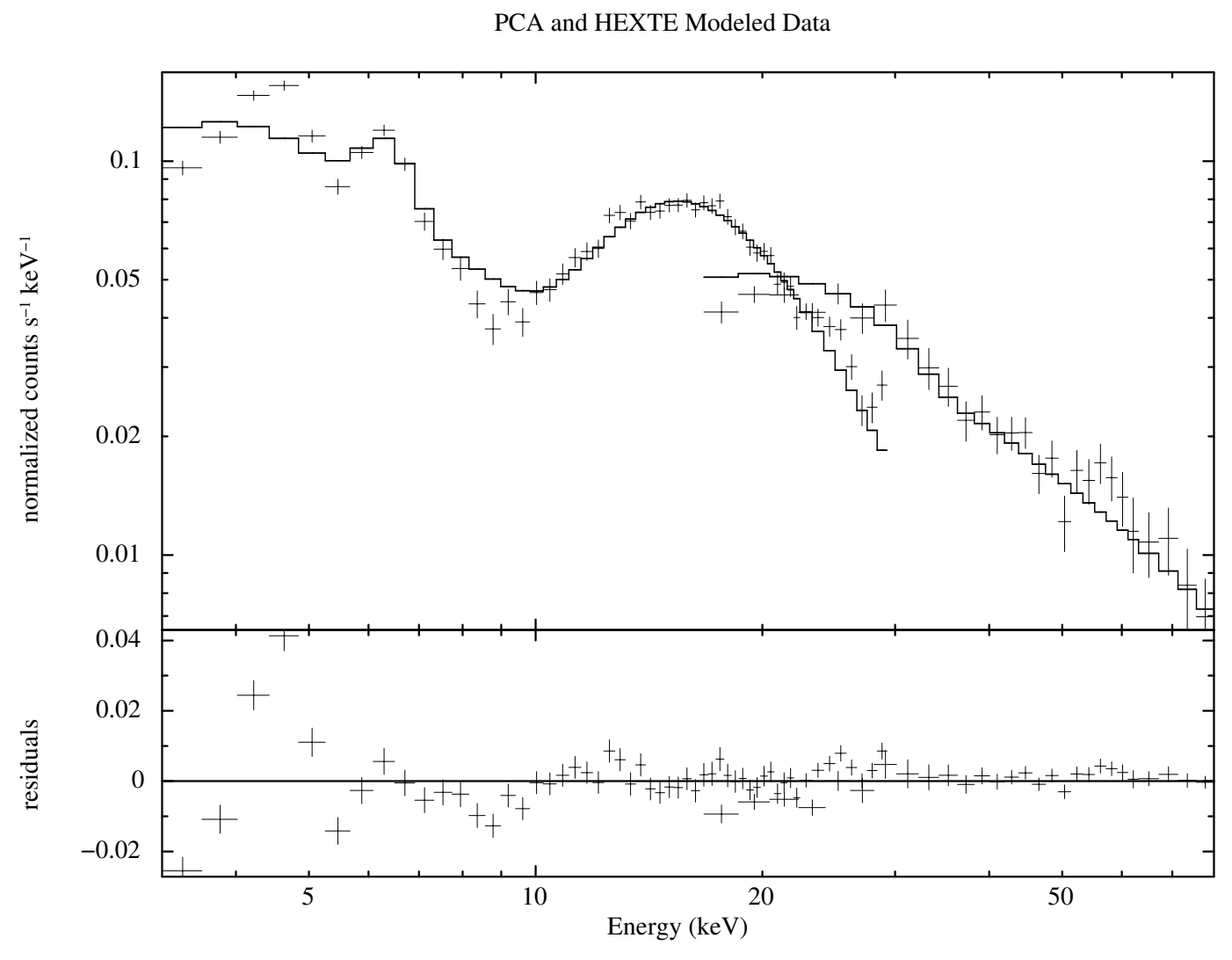

amiller 9-Aug-2007 13:36

Figure 4: Both PCA and HEXTE data shown with residuals corresponding to the model applied. The solid black line is the model while the crosses are data points (error bars included). The first unbroken segment in the energy range of 3-30 keV is from the PCA data, while the second segment in the energy range of $\sim 15-70 \mathrm{keV}$ is from the HEXTE data. The specifics of the model are discussed in the Results section. 
Unfolded Spectrum of PCA and HEXTE data

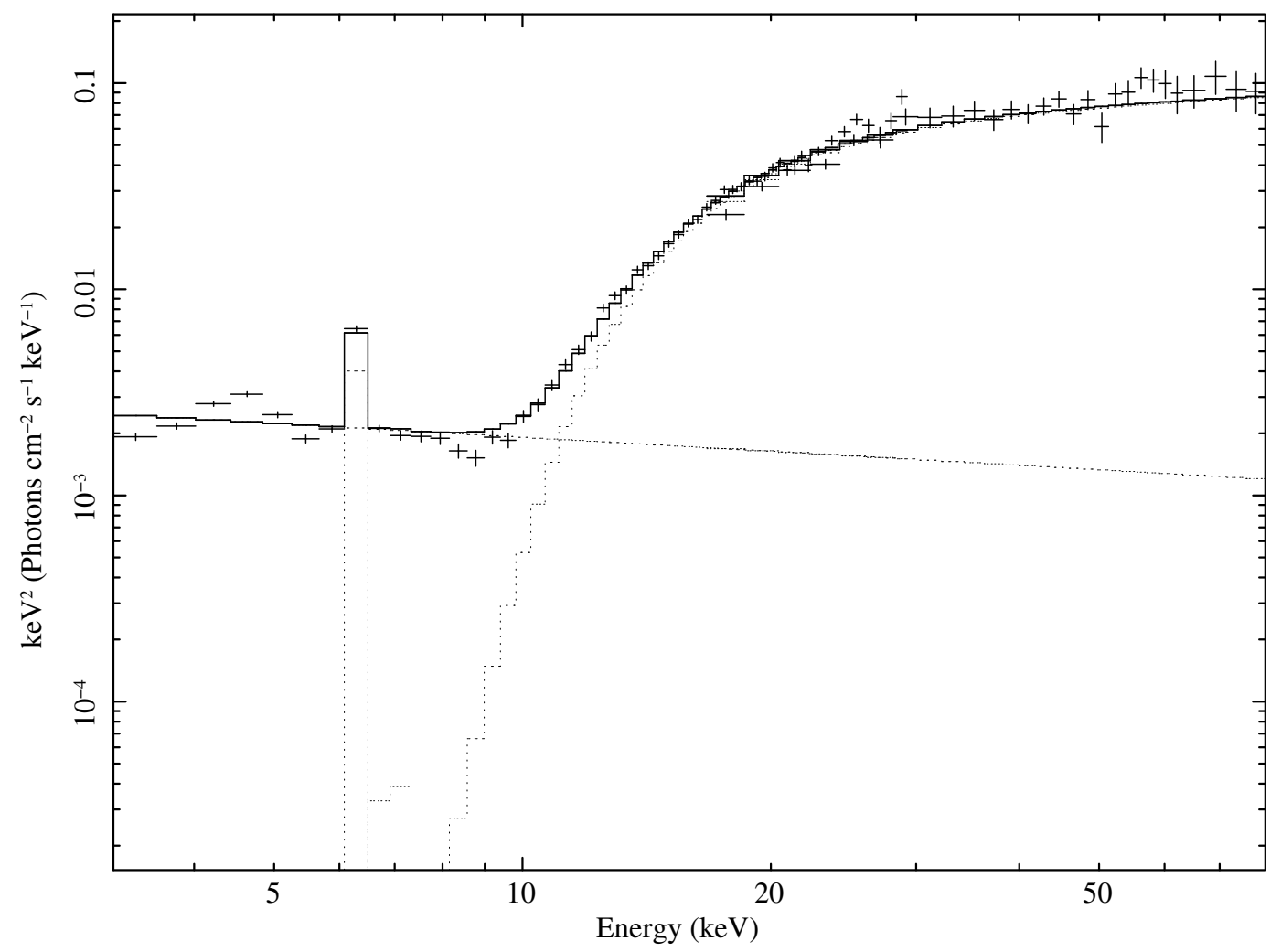

amiller 24-Jul-2007 11:21

Figure 5: PCA and HEXTE data on a logarithmic scale with the model used in Figure 4 (described in the Results section). The model is again the solid black line, while the crosses are data points (error bars included). The PCA data ranges from 3-30 keV, while the HEXTE data ranges from $\sim 15-80 \mathrm{keV}$. The HEXTE data and PCA correspond nicely to the model, with not obvious jumps or gaps, implying the data reduction process was successful. 


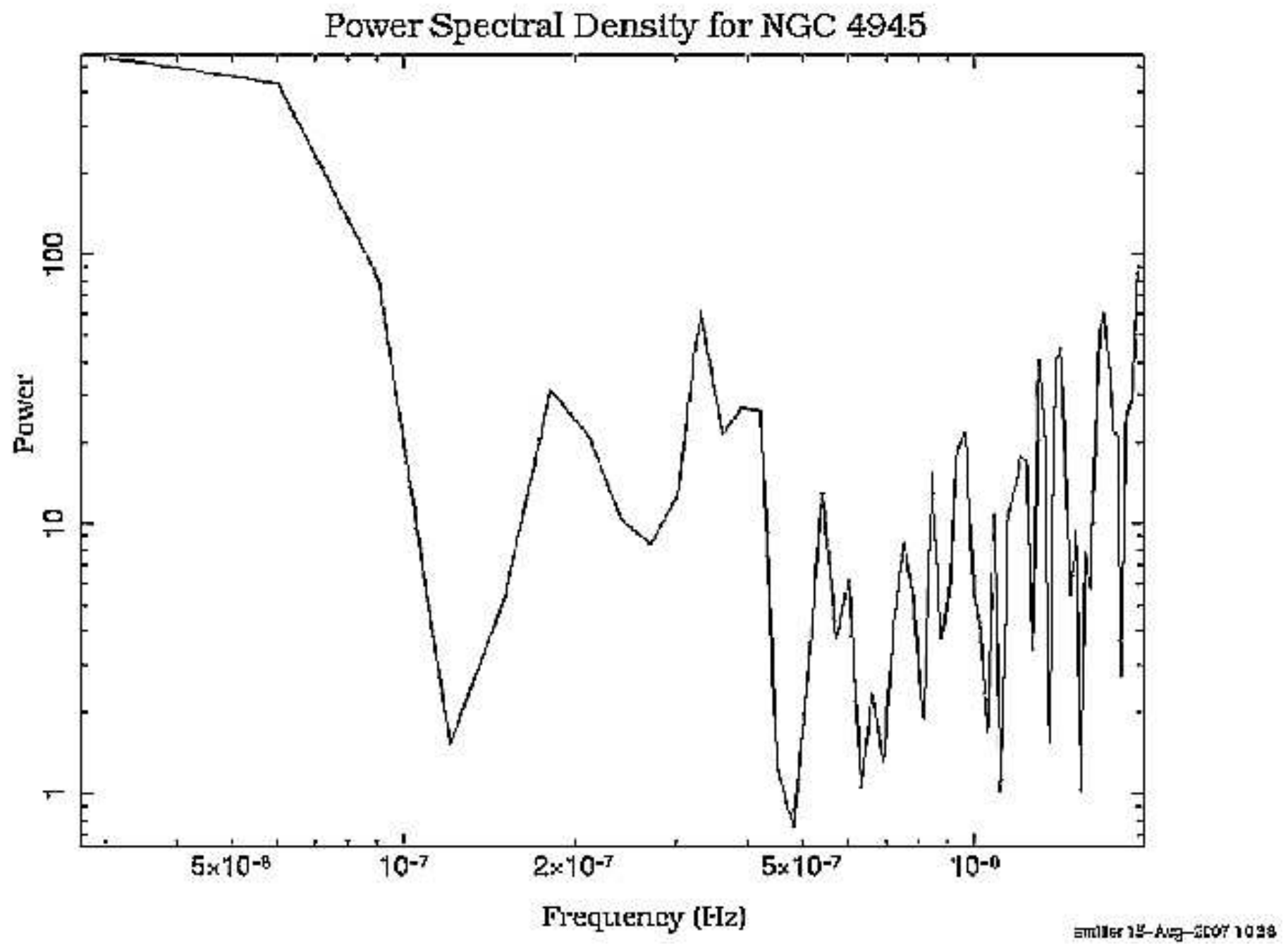

Figure 6: A PSD produced from PCA data. The scale is logarithmic, with Power as a function of Frequency. 


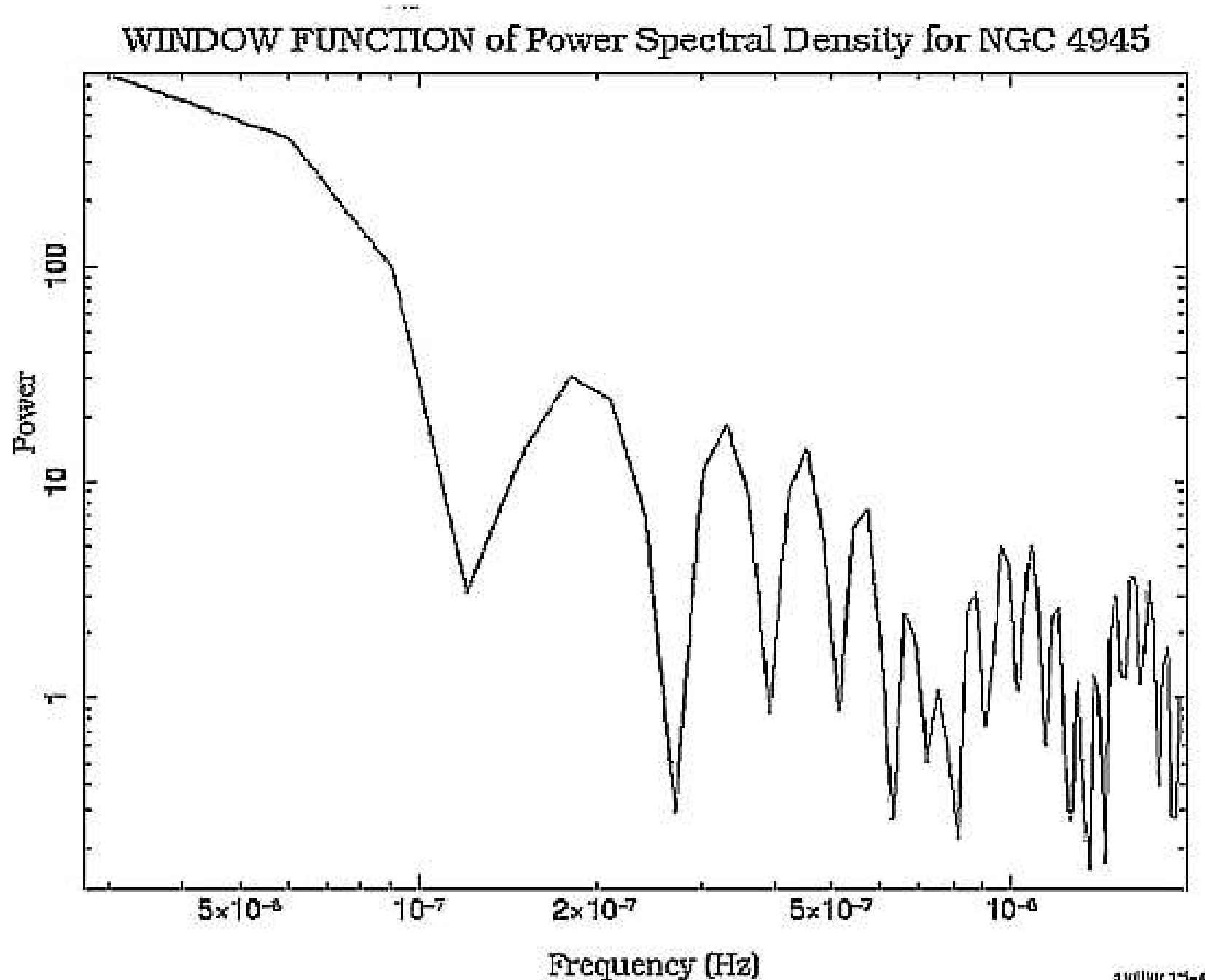

Figure 7: The Window Function for the PSD in Figure 6. The Window Function can be thought of as the intrinsic error of the FFT. The PCA data used in the FFT was analyzed and a blank copy of the pattern of that data was reproduced and run through an FFT, producing a "control" PSD to compare to the real PSD. The scale is also logarithmic. 